Physics in Collision (PIC 2013)

International Journal of Modern Physics: Conference Series

Vol. 31 (2014) 1460281 (7 pages)

(C) The Author

DOI: $10.1142 / \mathrm{S} 2010194514602816$

\title{
Applications of higher order QCD
}

\author{
Stefan Höche \\ SLAC National Accelerator Laboratory, \\ Menlo Park, CA 94025, USA \\ shoeche@slac.stanford.edu \\ Published 15 May 2014
}

In this talk we summarize some recent developments in perturbative QCD and their application to particle physics phenomenology.

\section{Introduction}

With the discovery of a new boson at the Large Hadron Collider (LHC), particle physics has entered a new era. Since this discovery, the field has quickly moved towards precision measurements on the new particle. In order to further improve these measurements and to find possible small deviations that may hint towards new physics, improved theoretical predictions, including higher-order perturbative QCD corrections for production rates and kinematics are urgently needed. The same is true for other reactions of interest at the LHC, like top quark production and $W / Z$ production. The toolkit used to this end ranges from fixed order calculations at the parton-level over resummation to parton showers and particle-level event generators. Tremendous progress has been made in the field during the past year. Some of the recent developments will be briefly summarized in this talk.

\section{Higher-Order Calculations}

Fixed-order calculations are available for a large variety of processes. At the tree level, they have long been performed completely automatically using programs like ALPGEN, ${ }^{1}$ Amegic,$++{ }^{2}$ Comix, ${ }^{3}$ CompHEP, ${ }^{4}$ HELAC, ${ }^{5}$ MadGraph $^{6}$ and Whizard. ${ }^{7}$ At the next-to-leading order (NLO), automation required two main ingredients: The implementation of known generic methods to perform the subtraction of infrared singularities, ${ }^{8-10}$ and the automated computation of one-loop amplitudes. As infrared subtraction terms consist of tree-level matrix elements joined

This is an Open Access article published by World Scientific Publishing Company. It is distributed under the terms of the Creative Commons Attribution 3.0 (CC-BY) License. Further distribution of this work is permitted, provided the original work is properly cited. 


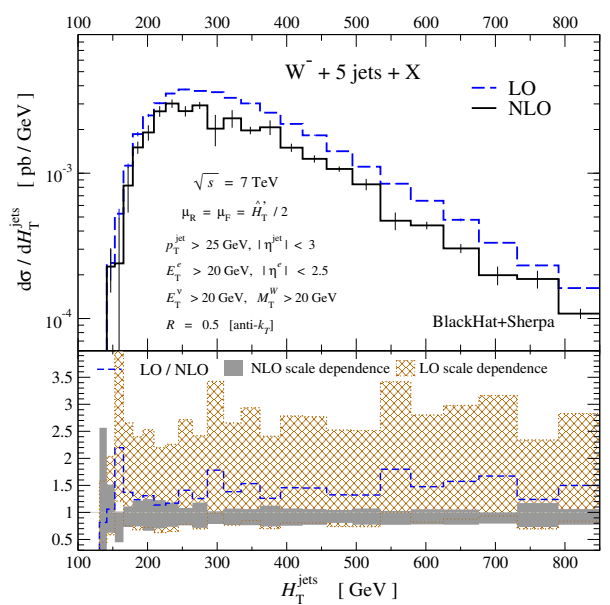

Fig. 1. Distribution of the visible energy in $W+5$ jet events. Figure taken from Ref. 16

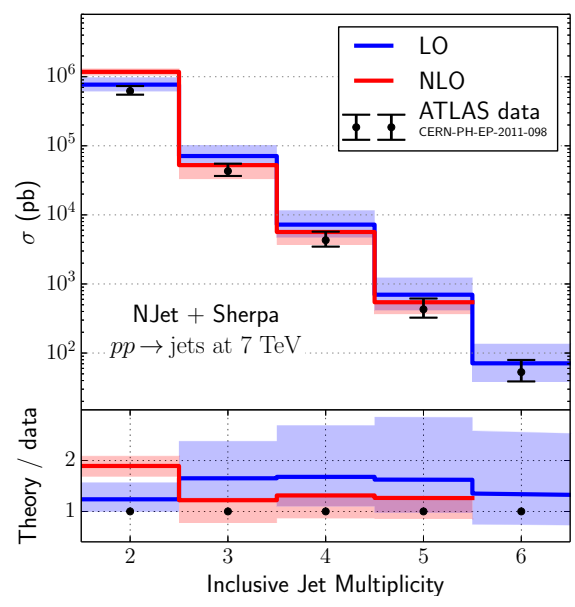

Fig. 2. Jet multiplicity distribution in pure jet events (right). Figure taken from Ref. 17.

by splitting operators, existing programs for leading order calculations are ideally suited to compute them. Correspondingly, Catani-Seymour dipole subtraction has been implemented in the existing generators Amegic,$++{ }^{11}$ Comix, HELAC ${ }^{12}$ and MadGraph. ${ }^{13,14}$ FKS subtraction is realized in MadGraph only. ${ }^{15}$

The automated computation of virtual corrections has received a boost from generalized unitarity ${ }^{18-20}$ which can be used to determine one-loop amplitudes by decomposing them into known scalar one-loop integrals and rational coefficients determined from tree amplitudes, plus a rational piece. ${ }^{21-25}$ Programs like BlackHat, ${ }^{26}$ Gosam, ${ }^{27}$ HELACNLO, ${ }^{28}$ MadLoop, ${ }^{29}$ NJet, ${ }^{30}$ OpenLoops ${ }^{31}$ and Rocket ${ }^{32,33}$ implement these techniques and supplement established programs like $\mathrm{MCFM}^{34,35}$ and dedicated codes based on improved tensor reduction approaches. ${ }^{36,37}$ New techniques have also been proposed to accelerate the numerical calculation of the integrand of one-loop amplitudes, independent of the reduction scheme. ${ }^{31}$ Figures 1 and 2 show examples from recent NLO calculations for $W+5$ jet production ${ }^{16}$ and 5 jets production, ${ }^{17}$ both performed using unitarity based techniques. Other recently completed calculations include Higgs boson plus 3 jet production ${ }^{38}$ and di-photon plus 2 jet production. ${ }^{39}$ The rapid progress in this field is reflected by the fact that all calculations from the experimenter's wishlist for the LHC have now been tackled. ${ }^{40}$ Most of the programs used to perform the calculations, or their results, are publicly available.

Driven by the need for higher precision in some selected Standard-Model reactions, the field of next-to-next-to leading order (NNLO) calculations has significantly advanced in the past years. One of the most challenging problems is the regularization of infrared divergences at NNLO. Sector decomposition ${ }^{41-43}$ has been used in the past to perform several $2 \rightarrow 1$ calculations. ${ }^{44,45}$ Antenna subtraction ${ }^{46,47}$ was worked out and implemented for $e^{+} e^{-} \rightarrow 3$ jets. ${ }^{48,49} q_{T}$ subtraction ${ }^{50}$ was employed 


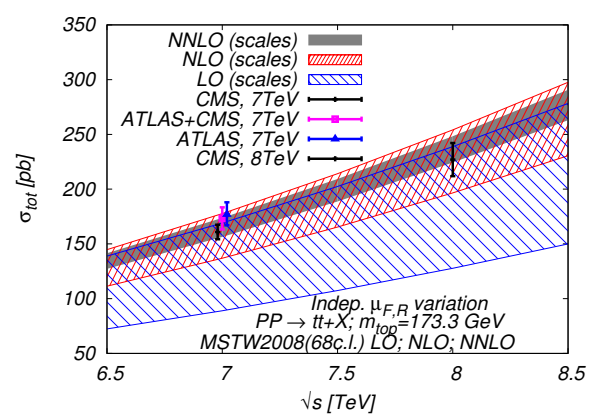

Fig. 3. Energy dependence of the $p p \rightarrow t \bar{t}$ total cross. Figure taken from Ref. 58.

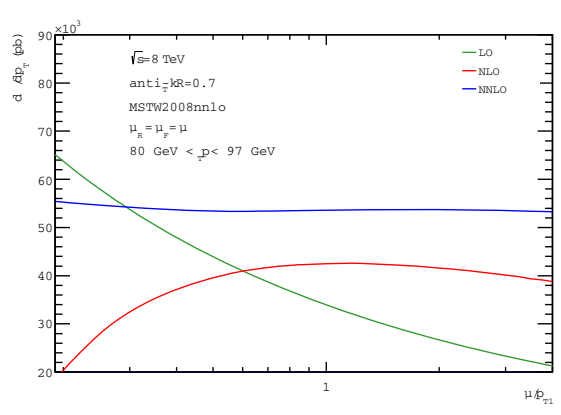

Fig. 4. Scale dependence of the $p p \rightarrow$ di-jet cross section. Figure taken from Ref. 64 .

in several calculations, including Higgs production, ${ }^{51} \mathrm{~W} / \mathrm{Z}$ production, ${ }^{52}$ associated Higgs production ${ }^{53}$ and di-photon production. ${ }^{54}$ More recently sector-improved subtraction methods were introduced. ${ }^{55,56}$ They have been used to compute cross sections for $p p \rightarrow t t^{57,58}$ and $p p \rightarrow H+$ jet. $^{59}$ At the same time, antenna subtraction was extended to initial states ${ }^{60-63}$ and employed to compute $p p \rightarrow$ di-jets fully differentially at NNLO. ${ }^{64}$ Figures 3 and 4 show results from some of these calculations. The calculation of $p p \rightarrow t \bar{t}$ has also been combined with higher logarithmic resummation. ${ }^{65-67}$ Its theoretical uncertainty is such that uncertainties from scale choices, PDF, strong coupling measurements and top-quark mass measurements are all of the same order. ${ }^{68}$

\section{Resummation of Jet Vetoes}

The analysis of the Higgs-like particle discovered at the LHC places new demands on resummed calculations. Many of the Higgs analysis channels, most notably $H \rightarrow$ $W W^{*} \rightarrow \ell^{+} \ell^{-} \nu \bar{\nu}$, veto on the transverse momentum of final state jets to distinguish different Standard Model backgrounds and separate them from the signal. The leading systematic uncertainty is the theoretical uncertainty on the signal cross section in the jet bins. This uncertainty can be reduced by a proper resummation of the logarithms associated with the jet veto. Various groups have investigated this problem, in most cases up to next-to-next-to leading logarithmic accuracy matched to NNLO fixed order, relying either on more traditional resummation methods, ${ }^{69,70}$ or on Soft Collinear Effective Theory. ${ }^{71-75}$ Higgs plus one jet production was studied at next-to-leading logarithmic order (NLL) and matched to NLO fixed order using SCET. ${ }^{76}$

\section{Parton Showers and Matching to NLO Calculations}

The interest in parton showers as a means to produce particle-level predictions fully differentially in the phase space of multi-jet events has increased significantly in 


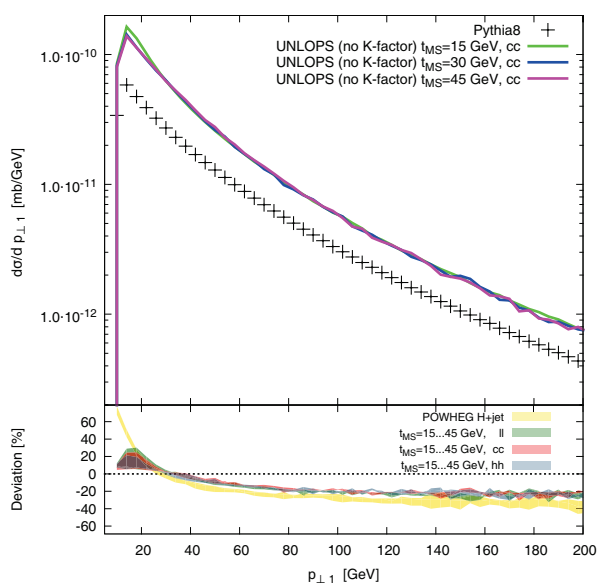

Fig. 5. Transverse momentum of first jet in Higgs plus jets events. Figure taken from Ref. 91.

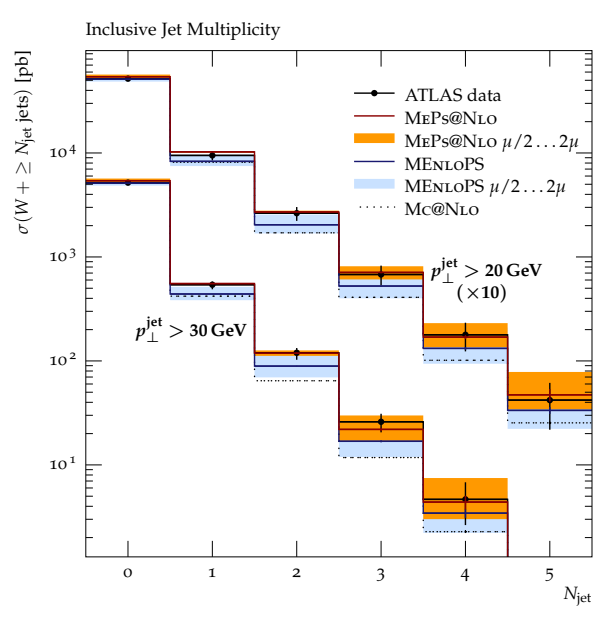

Fig. 6. Jet multiplicity distribution in $W+$ jets events. Figure taken from Ref. 92.

recent years. New concepts for the construction of parton showers have been proposed, which are based on antenna subtraction ${ }^{77,78}$ and/or sectorizing the phase space. ${ }^{79,80}$ Efforts were made to include subleading color corrections into showers as a means to improve their logarithmic accuracy. ${ }^{81,82}$ However, the crucial development was the proposal of a method to match parton showers to NLO calculations, ${ }^{83}$ later extended to eliminate negative weights. ${ }^{84,85}$ This matching has been partially or fully automated in several projects, ${ }^{86-90}$ such that particle-level predictions at NLO accuracy are now widely available.

The description of multi-jet final states with parton showers can be improved using so-called ME+PS merging methods, ${ }^{93-97}$ which, in contrast to matching methods, allow to correct the parton shower for an arbitrary number of emissions with higher-order tree-level calculations. These methods were recently refined and extended, leading to algorithms which can combine multiple NLO calculations of varying multiplicity (like $W+0$ jet, $W+1$ jet, $W+2$ jet, etc.) into a single, inclusive simulation (e.g. of $W+$ jets production). ${ }^{91,92,98-100}$ Figures 5 and 6 show examples for the application of ME+PS merging to Higgs boson plus jets production and to $W+$ jets production. A particular scale choice is required for the evaluation of the strong coupling in ME+PS merging, which has also been adopted for the matching to higher-multiplicity NLO calculations on its own in the so-called MINLO approach. ${ }^{101}$

The MINLO method accounts for Sudakov suppression effects in highermultiplicity final states and allows to extrapolate NLO calculations to zero jet transverse momentum, thus offering the opportunity to match to NNLO calculations for a limited class of processes and observables. ${ }^{102} \mathrm{~A}$ different proposal for a matching to NNLO parton-level calculations was made in, ${ }^{91,98}$ which is based on a subtraction method similar to the one used in ME+PS merging at NLO. Both techniques 
are promising candidates to further increase the precision of event generators for collider physics.

\section{Summary}

We have presented some of the recent developments in perturbative QCD and applications to particle physics phenomenology. NLO parton-level calculations can nowadays often be provided by fully automated tools. New techniques in event generation allow to also use them for particle-level predictions. NNLO calculations and higher-logarithmic resummation techniques are at the forefront of current research.

\section{References}

1. M. L. Mangano, M. Moretti, F. Piccinini, R. Pittau and A. D. Polosa, JHEP 07, p. 001 (2003).

2. F. Krauss, R. Kuhn and G. Soff, JHEP 02, p. 044 (2002).

3. T. Gleisberg and S. Höche, JHEP 12, p. 039 (2008).

4. E. Boos et al., Nucl. Instrum. Meth. A534, 250 (2004).

5. A. Kanaki and C. G. Papadopoulos, Comput. Phys. Commun. 132, 306 (2000).

6. J. Alwall, M. Herquet, F. Maltoni, O. Mattelaer and T. Stelzer, JHEP 06, p. 128 (2011).

7. W. Kilian, T. Ohl and J. Reuter, Eur. Phys. J. C71, p. 1742 (2007).

8. S. Frixione, Z. Kunszt and A. Signer, Nucl. Phys. B467, 399 (1996).

9. S. Catani and M. H. Seymour, Nucl. Phys. B485, 291 (1997).

10. S. Catani, S. Dittmaier, M. H. Seymour and Z. Trocsanyi, Nucl. Phys. B627, 189 (2002).

11. T. Gleisberg and F. Krauss, Eur. Phys. J. C53, 501 (2008).

12. M. Czakon, C. Papadopoulos and M. Worek, JHEP 08, p. 085 (2009).

13. R. Frederix, T. Gehrmann and N. Greiner, JHEP 09, p. 122 (2008).

14. R. Frederix, T. Gehrmann and N. Greiner, JHEP 06, p. 086 (2010).

15. R. Frederix, S. Frixione, F. Maltoni and T. Stelzer, JHEP 10, p. 003 (2009).

16. Z. Bern, L. Dixon, F. Febres Cordero, S. Hoeche, H. Ita et al., Phys. Rev. D88, p. 014025 (2013).

17. S. Badger, B. Biedermann, P. Uwer and V. Yundin (2013).

18. Z. Bern, L. J. Dixon, D. C. Dunbar and D. A. Kosower, Nucl. Phys. B435, 59 (1995).

19. Z. Bern, L. J. Dixon, D. C. Dunbar and D. A. Kosower, Nucl. Phys. B425, 217 (1994).

20. Z. Bern, L. J. Dixon and D. A. Kosower, Nucl. Phys. B513, 3 (1998).

21. G. Ossola, C. G. Papadopoulos and R. Pittau, Nucl. Phys. B763, 147 (2007).

22. D. Forde, Phys. Rev. D75, p. 125019 (2007).

23. R. Ellis, W. Giele and Z. Kunszt, JHEP 0803, p. 003 (2008).

24. G. Ossola, C. G. Papadopoulos and R. Pittau, JHEP 05, p. 004 (2008).

25. R. Ellis, W. T. Giele, Z. Kunszt and K. Melnikov, Nucl. Phys. B822, 270 (2009).

26. C. F. Berger, Z. Bern, L. J. Dixon, F. Febres-Cordero, D. Forde, H. Ita, D. A. Kosower and D. Maître, Phys. Rev. D78, p. 036003 (2008).

27. G. Cullen, N. Greiner, G. Heinrich, G. Luisoni, P. Mastrolia, G. Ossola and T. Reiter (2011).

28. G. Bevilacqua, M. Czakon, M. Garzelli, A. van Hameren, A. Kardos et al., Comput. Phys. Commun. 184, 986 (2013). 
29. V. Hirschi, R. Frederix, S. Frixione, M. V. Garzelli, F. Maltoni and R. Pittau, JHEP 1105, p. 044 (2011).

30. S. Badger, B. Biedermann and P. Uwer, Comput. Phys. Commun. 182, 1674 (2011).

31. F. Cascioli, P. Maierhofer and S. Pozzorini, Eur. Phys. J. C72, p. 1889 (2012).

32. R. Ellis, W. Giele, Z. Kunszt, K. Melnikov and G. Zanderighi, JHEP 0901, p. 012 (2009).

33. R. Ellis, K. Melnikov and G. Zanderighi, JHEP 0904, p. 077 (2009).

34. J. Campbell, R. K. Ellis and C. Williams.

35. J. M. Campbell and R. Ellis, Nucl. Phys. Proc. Suppl. 205-206, 10 (2010).

36. A. Denner and S. Dittmaier, Nucl. Phys. B734, 62 (2006).

37. T. Binoth, J. P. Guillet, G. Heinrich, E. Pilon and C. Schubert, JHEP 0510, p. 015 (2005).

38. G. Cullen, H. van Deurzen, N. Greiner, G. Luisoni, P. Mastrolia et al., Phys. Rev. Lett. 111, p. 131801 (2013).

39. T. Gehrmann, N. Greiner and G. Heinrich (2013).

40. J. Alcaraz Maestre et al. (2012).

41. T. Binoth and G. Heinrich, Nucl. Phys. B585, 741 (2000).

42. C. Anastasiou, K. Melnikov and F. Petriello, Phys. Rev. D69, p. 076010 (2004).

43. T. Binoth and G. Heinrich, Nucl. Phys. B693, 134 (2004).

44. C. Anastasiou, K. Melnikov and F. Petriello, Phys. Rev. Lett. 93, p. 262002 (2004).

45. K. Melnikov and F. Petriello, Phys. Rev. Lett. 96, p. 231803 (2006).

46. D. A. Kosower, Phys. Rev. D57, 5410 (1998).

47. A. Gehrmann-De Ridder, T. Gehrmann and E. W. N. Glover, JHEP 09, p. 056 (2005).

48. A. Gehrmann-De Ridder, T. Gehrmann, E. Glover and G. Heinrich, Phys. Rev. Lett. 99, p. 132002 (2007).

49. A. Gehrmann-De Ridder, T. Gehrmann, E. Glover and G. Heinrich, JHEP 0711, p. 058 (2007).

50. S. Catani and M. Grazzini, Phys. Rev. Lett. 98, p. 222002 (2007).

51. M. Grazzini, JHEP 0802, p. 043 (2008).

52. S. Catani, L. Cieri, G. Ferrera, D. de Florian and M. Grazzini, Phys. Rev. Lett. 103, p. 082001 (2009).

53. G. Ferrera, M. Grazzini and F. Tramontano, Phys. Rev. Lett. 107, p. 152003 (2011).

54. S. Catani, L. Cieri, D. de Florian, G. Ferrera and M. Grazzini (2011).

55. M. Czakon, Phys. Lett. B693, 259 (2010).

56. R. Boughezal, K. Melnikov and F. Petriello, Phys. Rev. D85, p. 034025 (2012).

57. P. Bärnreuther, M. Czakon and A. Mitov, Phys. Rev. Lett. 109, p. 132001 (2012).

58. M. Czakon, P. Fiedler and A. Mitov, Phys. Rev. Lett. 110, p. 252004 (2013).

59. R. Boughezal, F. Caola, K. Melnikov, F. Petriello and M. Schulze (2013).

60. A. Daleo, A. Gehrmann-De Ridder, T. Gehrmann and G. Luisoni, JHEP 1001, p. 118 (2010).

61. R. Boughezal, A. Gehrmann-De Ridder and M. Ritzmann, JHEP 1102, p. 098 (2011).

62. T. Gehrmann and P. F. Monni, JHEP 1112, p. 049 (2011).

63. A. Gehrmann-De Ridder, T. Gehrmann and M. Ritzmann, JHEP 1210, p. 047 (2012).

64. A. Gehrmann-De Ridder, T. Gehrmann, E. Glover and J. Pires, Phys. Rev. Lett. 110, p. 162003 (2013).

65. M. Czakon, A. Mitov and G. F. Sterman, Phys. Rev. D80, p. 074017 (2009).

66. M. Beneke, M. Czakon, P. Falgari, A. Mitov and C. Schwinn, Phys. Lett. B690, 483 (2010). 
67. M. Cacciari, M. Czakon, M. Mangano, A. Mitov and P. Nason, Phys. Lett. B710, $612(2012)$.

68. M. Czakon, M. L. Mangano, A. Mitov and J. Rojo, JHEP 1307, p. 167 (2013).

69. A. Banfi, G. P. Salam and G. Zanderighi, JHEP 1206, p. 159 (2012).

70. A. Banfi, P. F. Monni, G. P. Salam and G. Zanderighi, Phys. Rev. Lett. 109, p. 202001 (2012).

71. F. J. Tackmann, J. R. Walsh and S. Zuberi, Phys. Rev. D86, p. 053011 (2012).

72. I. W. Stewart, F. J. Tackmann, J. R. Walsh and S. Zuberi (2013).

73. T. Becher and M. Neubert, JHEP 1207, p. 108 (2012).

74. T. Becher, M. Neubert and D. Wilhelm, JHEP 1305, p. 110 (2013).

75. T. Becher, M. Neubert and L. Rothen, JHEP 1310, p. 125 (2013).

76. X. Liu and F. Petriello, Phys. Rev. D87, p. 094027 (2013).

77. W. T. Giele, D. A. Kosower and P. Z. Skands, Phys. Rev. D78, p. 014026 (2008).

78. L. Hartgring, E. Laenen and P. Skands, JHEP 1310, p. 127 (2013).

79. A. J. Larkoski and M. E. Peskin, Phys. Rev. D81, p. 054010 (2010).

80. A. J. Larkoski and M. E. Peskin, Phys. Rev. D84, p. 034034 (2011).

81. S. Plätzer and M. Sjödahl, JHEP 1207, p. 042 (2012).

82. S. Höche, F. Krauss, M. Schönherr and F. Siegert, JHEP 09, p. 049 (2012).

83. S. Frixione and B. R. Webber, JHEP 06, p. 029 (2002).

84. P. Nason, JHEP 11, p. 040 (2004).

85. S. Frixione, P. Nason and C. Oleari, JHEP 11, p. 070 (2007).

86. S. Alioli, P. Nason, C. Oleari and E. Re, JHEP 06, p. 043 (2010).

87. S. Hoche, F. Krauss, M. Schonherr and F. Siegert, JHEP 1104, p. 024 (2011).

88. R. Frederix, S. Frixione, V. Hirschi, F. Maltoni, R. Pittau et al., JHEP 1202, p. 048 (2012).

89. S. Höche, F. Krauss, M. Schönherr and F. Siegert, Phys. Rev. Lett. 110, p. 052001 (2013).

90. S. Höche and M. Schönherr, Phys. Rev. D86, p. 094042 (2012).

91. L. Lönnblad and S. Prestel, JHEP 1303, p. 166 (2013).

92. S. Höche, F. Krauss, M. Schönherr and F. Siegert (2012).

93. S. Catani, F. Krauss, R. Kuhn and B. R. Webber, JHEP 11, p. 063 (2001).

94. F. Krauss, JHEP 0208, p. 015 (2002).

95. M. L. Mangano, M. Moretti and R. Pittau, Nucl. Phys. B632, 343 (2002).

96. J. Alwall et al., Eur. Phys. J. C53, 473 (2008).

97. L. Lönnblad and S. Prestel, JHEP 1302, p. 094 (2013).

98. N. Lavesson and L. Lönnblad, JHEP 12, p. 070 (2008).

99. T. Gehrmann, S. Höche, F. Krauss, M. Schönherr and F. Siegert, JHEP 1301, p. 144 (2013).

100. R. Frederix and S. Frixione, JHEP 1212, p. 061 (2012).

101. K. Hamilton, P. Nason and G. Zanderighi (2012).

102. K. Hamilton, P. Nason, C. Oleari and G. Zanderighi, JHEP 1305, p. 082 (2013). 